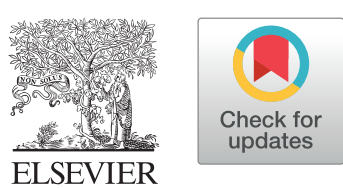

\author{
CJC Open 3 (2021) 684-686 \\ Case Report

\section{ALCAPA and ASD Secundum Unmasked by Severe Postpartum Haemorrhage Complicated by Heart Failure} \\ Siddarth Nosib, BSc, ${ }^{a}$ and Shravan Nosib, MD, FRCPC ${ }^{\text {b }}$ \\ ${ }^{a}$ Faculty of Medicine, University of Central Lancashire, Preston, United Kingdom \\ ${ }^{b}$ Department of Medicine, Royal University Hospital, University of Saskatchewan, Saskatoon, Saskatchewan, Canada
}

\begin{abstract}
Abnormal Left Coronary Artery from Pulmonary Artery (ALCAPA) is a rare congenital coronary anomaly in which the left main coronary artery arises from the pulmonary trunk, resulting in a left to right shunt. ALCAPA is associated with septal defects and patent ductus arteriosus. The case discussed had a secundum atrial septal defect. Sudden cardiac death is a feared complication. We discuss the hemodynamics of this shunt combination and outline its management, emphasizing shunt hemodynamics during pregnancy. This case highlights that pregnant patients with ALCAPA are at high risk and require optimal peri- and postpartum care. After surgical establishment of dual coronary circulation, patients have carried subsequent pregnancies to term.
\end{abstract}

\section{Case Presentation}

A previously healthy 18 -year-old female patient presented with chest pain syndrome after a normal vaginal delivery complicated by postpartum haemorrhage and a haemoglobin of $47 \mathrm{~g} / \mathrm{dL}$. Her blood pressure was $96 / 50 \mathrm{~mm}$ $\mathrm{Hg}$; she was tachycardic with a resting heart rate of $140 /$ min. She was in mild congestive heart failure with bilateral crackles at the lung bases. Cardiac auscultation was remarkable for a grade $3 / 6$ holosystolic murmur of mitral regurgitation at the apex. There was a prominent S3. Electrocardiogram showed diffuse ST-segment depression in the anterior leads. There were small Q waves in leads V1 and V2. Biomarkers confirmed myocardial ischemia. Troponins peaked at $230 \mathrm{ng} / \mathrm{L}$ with a brain natriuetic peptide of

Received for publication September 10, 2020. Accepted December 20, 2020.

Ethics Statement: We have adhered to the relevant ethical guidelines while writing this case report.

Corresponding author: Dr Shravan Nosib, Royal University Hospital, University of Saskatchewan, 1010 Pohorecky Bay Evergreen, Saskatoon, SK S7W0J4, Canada. Fax: 3069526115.

E-mail: shravan.nosib@gmail.com

See page 686 for disclosure information.

\begin{abstract}
RÉSUMÉ
L'ALCAPA est une anomalie coronarienne congénitale rare caractérisée par la naissance de l'artère coronaire gauche du tronc pulmonaire, qui entraîne un shunt gauche-droit. L'ALCAPA est associée à des malformations septales et une persistance du canal artériel. Le cas sur lequel nous nous sommes penchés avait une communication interauriculaire (CIA) de type ostium secundum. La mort subite d'origine cardiaque est la complication redoutée. Nous nous penchons sur I'hémodynamique de la combinaison de ce shunt et décrivons sa prise en charge, notamment celle de l'hémodynamique du shunt au cours de la grossesse. Ce cas illustre que les patientes enceintes qui ont une ALCAPA sont exposées à un risque élevé et qu'elles ont besoin de soins optimaux avant et après l'accouchement. Après l'établissement chirurgical de la circulation coronarienne double, les patientes ont mené à terme les grossesses subséquentes.
\end{abstract}

$1800 \mathrm{ng} / \mathrm{L}$. Chest radiograph showed evidence of pulmonary oedema. She was stabilized in the coronary care unit.

Bedside echocardiography documented anterior wall hypokinesis and moderate left ventricular dysfunction with an estimated left ventricular ejection fraction of approximately $38 \%$. There was moderate mitral regurgitation. Abnormal flow was noted in the pulmonary artery $(\mathrm{PA})$ in the short-axis views, and prominent collaterals were seen in the interventricular septum (Fig. 1). A dilated right coronary artery (RCA) was also clearly visualized. The right atrium and right ventricle were normal in size. Right ventricular systolic pressure was approximately $28 \mathrm{~mm} \mathrm{Hg}$. Transoesophageal echocardiography confirmed these findings. A secundum atrial septal defect (ASD) $(10 \mathrm{~mm})$ was also noted. There was a left to right shunt. The Qp/Qs ratio was approximately $1.4 / 1$.

Abnormal origin of the left main coronary artery (LMCA) from the PA was considered, and the patient underwent cardiac catheterization. The LMCA originated from the main pulmonary trunk. The RCA was large and aberrant and provided collateral flow to the left coronary tree (Video 1 …이) . An oxygen saturation run showed step-up at the level of the main PA (right ventricle 67\%, PA 78\%). Shunt fraction was estimated at 


\section{Novel Teaching Points}

- ALCAPA is a rare congenital abnormality, the hemodynamic hallmark of which is a left to right shunt. If untreated, it has high lethality.

- It may be associated with septal defects and patent ductus arteriosus.

- Pregnancy in patients with ALCAPA is a high-risk scenario requiring optimal peri- and postpartum care as well as close hemodynamic monitoring for heart failure and arrhythmic SCD.

- Optimal management of ALCAPA is surgical with the establishment of a dual coronary circulation.

- Operated patients with ALCAPA have carried subsequent pregnancies to term.

66\%. Left ventricular end-diastolic pressure was estimated at 38 $\mathrm{mm} \mathrm{Hg}$. Right atrial pressure was $14 \mathrm{~mm} \mathrm{Hg}$, right ventricular pressures were $49 / 21 \mathrm{~mm} \mathrm{Hg}$, PA systolic pressures were approximately $47 \mathrm{~mm} \mathrm{Hg}$, and a mean pressure was approximately $25 \mathrm{~mm} \mathrm{Hg}$.

The patient underwent surgery after medical optimization; the LMCA was reimplanted into the aortic root, the PA was patched, and the ASD secundum was closed. Her postoperative course was complicated by spinal infarction at the T8 level. This was unexplained as there was no report of significant bleeding or hypotension in the operating room. Subsequent laminectomy was performed to release a tethered cord, unfortunately without any significant improvement.

When last seen the patient was doing well from the cardiovascular standpoint. Outpatient transthoracic echocardiography documented a left ventricular ejection fraction of $46 \%$ and mild-to-moderate mitral regurgitation.
Although she is confined to a wheelchair, she leads an active life and has recently carried a pregnancy to term. She is well compensated from the cardiovascular standpoint and has not had any admissions with heart failure exacerbations, more than 10 years after surgery.

\section{Discussion}

Anomalous origin of the left coronary artery from the PA, also known as abnormal left coronary artery from pulmonary artery (ALCAPA) or Bland-White-Garland syndrome, is an uncommon congenital coronary anomaly and is reported in approximately $0.26 \%$ of patients with congenital heart disease. $^{1}$

The fundamental hemodynamic abnormality in ALCAPA is a left to right shunt. With the fall in pulmonary vascular resistance at birth, a left to right shunt circuit is established with forward flow from the RCA to the left coronary artery to the PA. A steal syndrome ensues, thus exposing the anterior myocardial wall to ischemia. The development of a collateral network from the RCA is critical for maintaining anterior wall perfusion and preventing ischemic complications. Even so few patients survive past childhood without surgical repair and up to $90 \%$ die suddenly at a mean age of 35 years though there are case reports of patients surviving into their seventies. Physical examination of patients with ALCAPA may reveal a systolic murmur of ischemic mitral regurgitation and has been described in approximately $70 \%$ of patients. ${ }^{2}$ Impressive collateral flow may manifest as a continuous murmur.

Noninvasive diagnosis of ALCAPA can be challenging, but characteristic echocardiographic findings have been described in the literature. Extensive collaterals between right and left coronary arteries (Fig. 1, Video 1) have been described as an echocardiographic diagnostic marker for ALCAPA. The RCA is dilated and easily seen at transthoracic echocardiogram. ALCAPA is usually an isolated

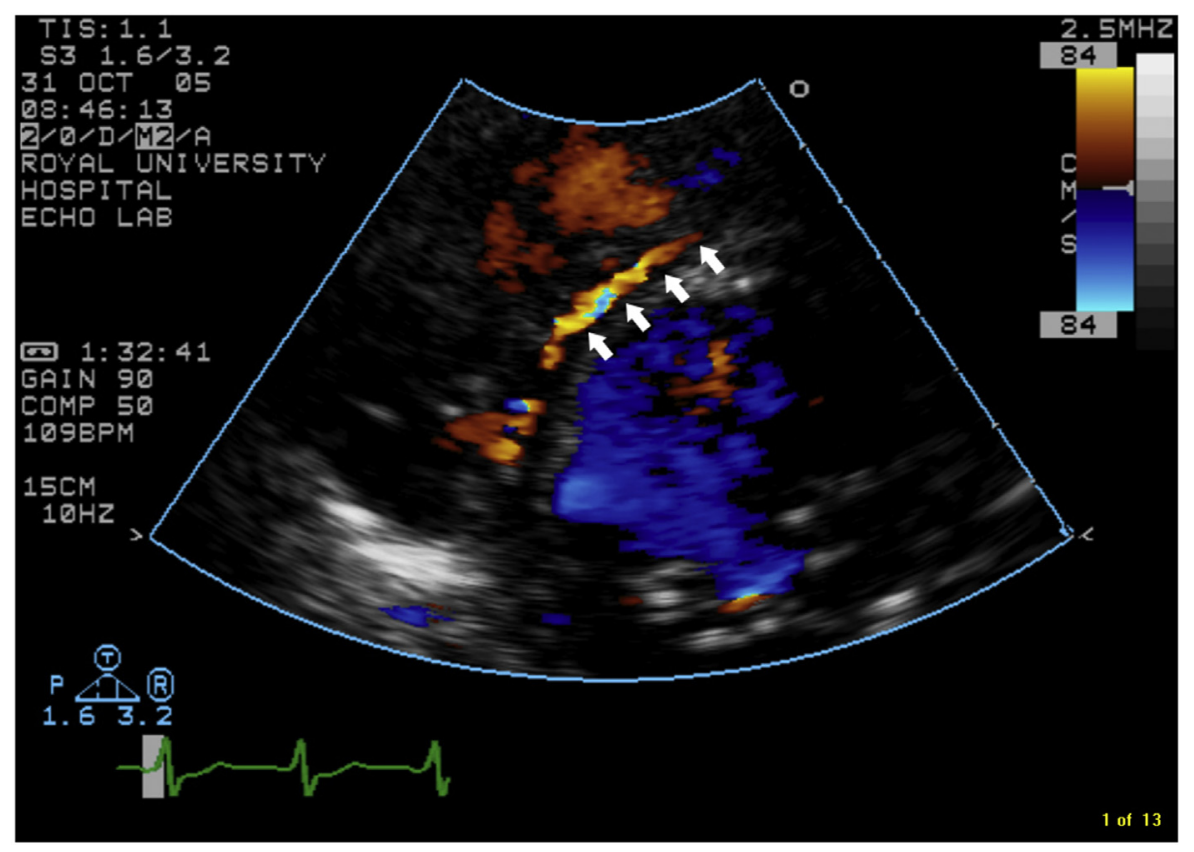

Figure 1. Short-axis view showing collaterals (arrows) in the interventricular septum. 
anomaly, but associations with patent ductus arteriosus, ventricular septal defect, tetralogy of Fallot, or coarctation of aorta have been reported in the literature and these should be sought for diligently at echocardiography. ${ }^{3}$

Computed tomography angiography and magnetic resonance imaging have improved the noninvasive diagnosis of ALCAPA. Potential advantages include direct visualization of the origin of the LMCA from the posterior aspect of the PA, dilatation and tortuosity of the RCA, and visualization of dilated intercoronary collateral arteries along the external surface of the heart or within the interventricular septum. ${ }^{4}$ Conventional angiography remains the gold standard for the diagnosis of ALCAPA as for most other coronary artery anomalies, and it has the added advantage of defining the severity of ischemic mitral regurgitation as well as the degree of left ventricular dysfunction.

Preoperative imaging of the spinal cord with computed tomography/magnetic resonance imaging in patients with ALCAPA may help rule out congenital anomalies of the spinal cord and/or spinal circulation that may predispose these unfortunate patients to stroke peri- or postoperatively. Our patient developed a stroke postoperatively and was found to have tethering of the cord.

The concurrence of an ASD with ALCAPA is extremely rare with only a couple of cases reported in the literature. It may muddy the diagnostic waters further and pose therapeutic challenges if the shunt is significant. A significant shunt across the ASD, with increased flow and oxygen saturation in the pulmonary circuit, may improve blood and oxygen supply to the left coronary system by attenuating left coronary flow, especially with the development of pulmonary hypertension. This may have been the case in our patient as she had been minimally symptomatic throughout her pregnancy. Postpartum haemorrhage was, for her, a blessing in disguise. Percutaneous or surgical closure of the ASD, without knowledge of coronary anatomy, could potentially entail disastrous hemodynamic consequences due to loss of bidirectional shunting.

Simsek et al. ${ }^{5}$ have reported on a 32-year-old patient with ALCAPA who had carried 3 uncomplicated pregnancies to term without any complications during delivery or during the postpartum period. She had presented with chest pain syndrome and after diagnosis had undergone corrective surgery. They theorize that despite the hypervolemia and increased cardiac output of pregnancy, the patient remained asymptomatic through 3 pregnancies due to an optimal balance between the dilated and tortuous RCA and left coronary system via well-established collaterals. Her presenting symptoms were probably related to partial failure of this collateral circulation. ${ }^{5}$ Hence, collateral support remains critical in these patients to maintain adequate perfusion of the anterior myocardial wall. This mechanism may explain why our patient was minimally symptomatic during her pregnancy.

Definitive management of ALCAPA is surgical with a view to establish a dual coronary system. Surgical procedures include coronary artery bypass grafting, coronary baffling procedures, and direct reimplantation of the left coronary artery to the aorta. ${ }^{6}$ Cardiac transplantation has been reportedly performed in patients with severe left ventricular dysfunction at presentation. However, the series by Schwartz et al.,' in which they reviewed 42 patients with ALCAPA, concluded that the current operative survival for ALCAPA does not justify consideration of a major undertaking such as cardiac transplantation. Normalization of left ventricular systolic function is also expected in all operative survivors with a patent dual coronary system as per their finding. Interestingly, in the same series, the severity of preoperative mitral regurgitation was a prognostic determinant, whereas the severity of preoperative left ventricular systolic dysfunction and the degree of left ventricular remodelling were not.

\section{Conclusion}

ALCAPA is a rare congenital coronary anomaly with high lethality. The hemodynamic hallmark is a left to right shunt that may cause myocardial ischemia through various mechanisms, mainly failure of collateralization, compromise of the right coronary system, or severe anaemia from acute blood loss as discussed in our patient. Pregnancy in such patients is fraught with potential hemodynamic catastrophes. Arrhythmic SCD is a dreaded complication. Management is surgical. Patients have carried successful pregnancies to term after repair.

\section{Funding Sources}

No funding was received for this study.

\section{Disclosures}

The authors have no conflicts of interest to disclose.

\section{References}

1. Maeder M, Vogt PR, Amman P, Rickli H. Bland-White-Garland syndrome in a 39-year-old mother. Ann Thorac Surg 2004;78:1451-3.

2. Wilson CL, Dlabal PW, Holeyfiled RW, Akins CW, Knauf DG. Anomalous origin of left coronary artery from pulmonary artery-case report and review of the literature concerning teenagers and adults. J Thorac Cardiovasc Surg 1977;73:887-93.

3. Rao BNS, Lucas RV Jr, Edwards JE. Anomalous origin of the left coronary artery from the right pulmonary artery associated with ventricular septal defect. Chest 1970;59:616-20.

4. Khanna A, Torigian DA, Ferrari VA, Bross RJ, Rosen MA. ALCAPA in adulthood on CT and MRI. AJR Am J Roentgenol 2005;185:326-9.

5. Simsek H, Tuncer M, Yaman M, Celik M. Presentation of adult BWG syndrome in a 32 year old female. Int J Cardiovasc Acad 2015;1:56-8.

6. Vouhe PR, Tamisier D, Sidi D, et al. Anomalous left coronary artery from the pulmonary artery: results of isolated aortic reimplantation. Ann Thorac Surg 1992;54:621-7.

7. Schwartz ML, Jonas RA, Colan SD. ALCAPA: recovery of left ventricular function after dual coronary repair. J Am Coll Cardiol 1997;30:547-53.

\section{Supplementary Material}

To access the supplementary material accompanying this article, visit CJC Open at https://www.cjcopen.ca/ and at https://doi.org/10.1016/j.cjco.2020.12.016. 